\title{
A Letter Regarding Recently Published Original Article: Comparative Evaluation of Intraoral and Extraoral Periapical Radiographic Techniques in Determination of Working Length: An In Vivo Study
}

Johan KM Aps

International Journal of Clinical Pediatric Dentistry (2020): 10.5005/jp-journals-10005-1845

Dear Dr Nikhil Marwah,

Editor-in-Chief, International Journal of Clinical Pediatric Dentistry,

I am writing you to express my concerns regarding the following paper: Sridhara A, Konde S, Noojadi SR, et al. Comparative Evaluation of Intraoral and Extraoral Periapical Radiographic Techniques in Determination of Working Length: An In Vivo Study. Int J Clin Pediatr Dent 2020;13(3):211-216.

As a pediatric dentist and dental and maxillofacial radiologist, I was eager to read the paper by Sridhara et al. in your distinguished journal. The technique of extraoral periapicals is not new but very interesting and a good "plan B" in certain circumstances, as was mentioned in the literature by others. ${ }^{1-3}$ However, when I read the "Materials and Methods" section, I felt the urge to write this letter to the editor, as I wish to express my concerns about the "do no harm" principle and the patient's radiation safety. I wish to emphasize that my concerns are to be considered constructive and in no way I am trying to offend Sridhara et al.

The authors had selected patients, aged 13-25 years of age, who required exodontia of the first premolars for orthodontic purposes. The patients were subsequently subjected to unnecessary dental surgery (endodontic treatment) and unnecessary radiographic examinations, which included at least one intraoral periapical radiograph and one extraoral periapical radiograph. Nothing was mentioned about how many retakes were needed. However, I am certain they were not $100 \%$ successful in every case. Finally the researchers extracted these "for no reason endodontically treated teeth" for further research purposes, namely a comparison of the accuracy of the two radiographic techniques. In my perception, this study protocol has breached several ethical standard principles.

According to the American Dental Association (ADA: www.ADA. org/ethics), ethics in dentistry consists of five concepts: ${ }^{4}$

- Patient autonomy (patient self-governance)

- Nonmaleficence (do no harm)

- Beneficence (do good)

- Justice (be fair)

- Veracity (truthfulness)

The Federation Dentaire Internationale (FDI) or the World Dental Federation (www.fdiworlddental.org) has published similar guidelines. ${ }^{5}$ I feel the authors have, in their eagerness to pursue research, breached two concepts of the code of ethics: the nonmaleficence and the beneficence. There was a potential risk of
Department of Dentomaxillofacial Radiology, Artevelde University of Applied Sciences, Ghent, Belgium

Corresponding Author: Johan KM Aps, Department of Dentomaxillofacial Radiology, Artevelde University of Applied Sciences, Ghent, Belgium, Phone: +32 493 300289, e-mail: johan. apsdmfr@hotmail.com

How to cite this article: Aps JKM. A Letter Regarding Recently Published Original Article: Comparative Evaluation of Intraoral and Extraoral Periapical Radiographic Techniques in Determination of Working Length: An In Vivo Study. Int J Clin Pediatr Dent 2020;13(5):576-577.

Source of support: Nil

Conflict of interest: None

causing inflammatory reactions due to unnecessary endodontic treatment, caused by overinstrumentation and/or copious irrigation with $3 \%$ sodium hypochlorite, for instance. Additionally, there was the use of ionizing radiation, which carries a potential health risk for the patients (stochastic effects). With regard to the radiation exposure, it should be emphasized that since the three basic principles of radiation protection are justification, optimization, and limitation, one can easily understand my concern regarding the justification of these radiographs. ${ }^{6}$ The initial orthodontic decision was based on clinical and radiographic evidence and therefore no additional imaging was really required (no justification). Subsequently the two other principles were, unfortunately, breached as well.

The authors mention the use of a lead apron as being a measure to protect the patient. However, lead aprons are supposed to protect the patient from the primary radiation beam reaching unwanted anatomical regions (not the region of interest) and they do not protect patients from scattered radiation (inherent to the use of X-rays). Moreover, in Figures 1 and 2 of the paper, one can appreciate that the lead apron did not provide any thyroid gland protection, which somehow could have protected the (young) patients from scattered radiation toward the thyroid gland. ${ }^{7}$ The use of the apron was therefore useless and of no benefit. As a matter of fact, the scattered radiation from the extraoral periapical radiographs is much higher as the irradiated surface is larger, compared to the intraoral periapical radiographs..$^{8-10}$

The authors mention that patients signed an informed consent, but nowhere one can find evidence of an ethics committee involved in this study. This is raising concerns as one should certainly not use patients for research purposes if the research protocol itself is 
potentially harmful. A better approach would have been to perform exodontia of these first premolars as was intended to be done based on the orthodontic treatment plan. Subsequently, the researchers should have mounted the extracted teeth in a phantom, which would have allowed them to execute the endodontic treatment and take the radiographs they planned to compare. The latter would have been a safer and more ethical manner to conduct this research. The technique to obtain periapical radiographs with an extraoral approach certainly has applications in patients with special needs or in any circumstances where intraoral radiographs cannot be taken for a myriad of reasons (e.g., gagging, trismus). I applaud the efforts of the authors to publish about these undervalued techniques. However, the ethical issues of research protocols should not be ignored.

I hope this letter will engage researchers to be more serious about research ethics.

\section{References}

1. Fisher D. Extraoral radiographic technique of third molars. Aust Dent J 1974;19(5):306-307. DOI: 10.1111/j.1834-7819.1974.tb02812.x.

2. Sano K, Kitamori H, Ariji E, et al. Simple X-ray film holder for lateral view of the anterior jaws using dental X-ray apparatus. Br J Oral
Maxillofac Surg 1998;36(2):135-137. DOI: 10.1016/S0266-4356(98) 90182-6.

3. Newman ME, Friedman S. Extraoral radiographic technique: an alternative approach. JOE 2003;29(6):419-421.DOI: 10.1097/00004770200306000-00010.

4. www.ADA.org/ethics.

5. www.fdiworlddental.org.

6. International Commission on Radiological Protection. The 2007 recommendations of the international commission on radiological protection. Ann ICRP 2007;37(2-4):1-332. DOI: 10.1016/j. icrp.2007.11.001.

7. Han MA, Kim JH. Diagnostic X-ray exposure and thyroid cancer risk: systematic review and meta-analysis. Thyroid 2018(2):220-228. DOI: 10.1089/thy.2017.0159.

8. Tsapaki V. Radiation protection in dental radiology - recent advances and future directions. Phys Med 2017;44:222-226. DOI: 10.1016/j. ejmp.2017.07.018.

9. Hoogeveen R, Hazenoot B, Sanderink GCH, et al. The value of thyroid shielding in intraoral radiography. Dentomaxillofac Radiol 2016;45(5):20150407. DOI: 10.1259/dmfr.20150407.

10. Van Acker JWG, Pauwels NS, Cauwels RGEC, et al. Outcomes of different radioprotective precautions in children undergoing dental radiography: a systematic review. Eur Arch Paed Dent 2020;21(4):463508. DOI: $10.1007 / \mathrm{s} 40368-020-00544-8$. 